\title{
Influence of spatio-temporal heterogeneity on biogeochemical cycling in the subsurface using a numerical modeling approach
}

SWAMINI KHURANA ${ }^{1}$, FALK HEßE ${ }^{2}$, MARTIN ThULLNER

${ }^{1}$ Department of Environmental Microbiology, Helmholtz Center for Environmental Research - UFZ, Leipzig, 04318, Germany - swamini.khurana@ufz.de; martin.thullner@ufz.de

${ }^{2}$ Department of Computational Hydrosystems, Helmholtz Center for Environmental Research - UFZ, Leipzig, 04318, Germany - falk.hesse@ufz.de

Biogeochemical cycles impact most ecosystem functions by controlling the distribution of nutrients in an environmental compartment. The Critial Zone accounts for a large proportion of the global carbon and nitrogen budget, wherein microbes execute nearly all the natural processes contributing to biogeochemical cycles. The terrestrial subsurface is known to exhibit spatial heterogeneity and temporal dynamics leading to varying drivers of biogeochemical processes. Specifically, the shallow subsurface is impacted by temporal variations of water and solute fluxes linked with weather events. Hence, prediction of biogeochemical cycles in this compartment is a challenge due to the dynamic nature of this zone and limited observational opportunities. Therefore, the authors undertake a numerical modeling approach to study the impact of spatio-temporal heterogeneities on nutrient cycles in the subsurface.

An extended biogeochemical process network that accounted for autotrophic and heterotrophic microbial activity and associated life cycle processes such as respiration, growth, dormancy and mortality was set up. Several scenarios that described spatial and temporal heterogeneity were conceptualized, driven by data obtained from a subject site (AquaDiva Critical Zone Observatory, Hainich National Park, Thuringia, Germany [1]). Simulations investigating different combinations of these scenarios were carried out using OGSBRNS [2].

Results indicate that the flow regime and spatio-temporal heterogeneity impact redox dynamics, microbial activity and thereby nutrient cycling. These results aid in identification of drivers of microbial redox dynamics in the shallow subsurface, in turn assisting in upscaling of the process descriptions.

Refs:

[1] Küsel et al., 2016. Frontiers in Earth Science, 4: 32.

[2] Centler et al., 2010. Computers \& Geosci. 36: 397-405. 